\title{
Calibrating and testing of the forecasts of seismic hazard for planned mining sequences
}

\author{
DA Malovichko Institute of Mine Seismology, Australia
}

\begin{abstract}
The forecast of seismic hazard in mines depends on the planned mining sequence and therefore it is required to:

1. Model the changes in stresses and strains associated with future mining.

2. Transform these changes to the parameters of expected potentially damaging seismic events (location, time, size, mechanism).
\end{abstract}

This modelling of expected seismicity has to be calibrated. It needs to be shown that the recorded seismic response to the mining in the past can be replicated using a numerical model. The seismic hazard calculated for future mining steps also needs to be tested against the observed seismicity after the planned mining is completed.

The same mathematical framework can be used for both the calibration and the testing of seismic hazard forecasts. The area skill score (Zechar \& Jordan 2008) is adopted to assess the match between the location of significant seismic events and calculated hazard maps for the past mining steps (calibration) and forecast maps for the future mining steps (testing). The 3D rotation angle (Kagan 2007) is used to compare the source mechanisms of recorded significant seismic events with the expected mechanisms for the past and future mining steps. The seismic events affecting the poor performance of the forecast both in terms of location and source mechanisms can guide possible adjustment to the input parameters of the model (e.g. orientation of in situ stress, failure criteria) and help to improve the forecasts.

The suggested approach of calibrating and testing of seismic hazard forecasts is illustrated using data from Renison mine, Australia.

Keywords: modelling of seismicity, area skill score, 3D angle of earthquake double-couple rotation

\section{Introduction}

A genuine seismic event represents a sudden inelastic deformation within a given volume of rock that radiates detectable seismic waves (Mendecki 1997). Such a deformation can damage underground excavations if the latter is located within the deformed volume or strongly affected by the radiated transient stress pulse. To be able to manage these unfavourable scenarios, it is necessary to assess the seismic hazard by evaluating the probability of the occurrence of potentially damaging sudden deformation in a given volume and in a given period of time in the future. Subsequently, the calculated seismic hazard can be transferred to hazard in terms of parameters suitable for the design of excavations and support (e.g. the probability of exceedance of a potentially damaging level of dynamic tangential straining of the excavation walls or of a potentially damaging level of ground motion).

There are established techniques in global seismology to calculate the probabilities (or rate) of potentially damaging tectonic earthquakes in local areas. Usually these are based on the available catalogues of earthquakes (instrumental or historical). However, other information and methods are utilised as well (Field 2007) and these include slip rate for the mapped faults, strain rate in the earth crust reconstructed from GPS data, numerical modelling of stresses and unstable slip along faults (earthquake simulators). 
In mines, seismic hazard is typically related not to tectonic earthquakes, but to seismicity induced by mining. This implies that the recorded seismic data can be used only for short term forecasting of seismic hazard and that future mining needs to be taken into account for longer term forecasts. This aspect is depicted in Figure 1.

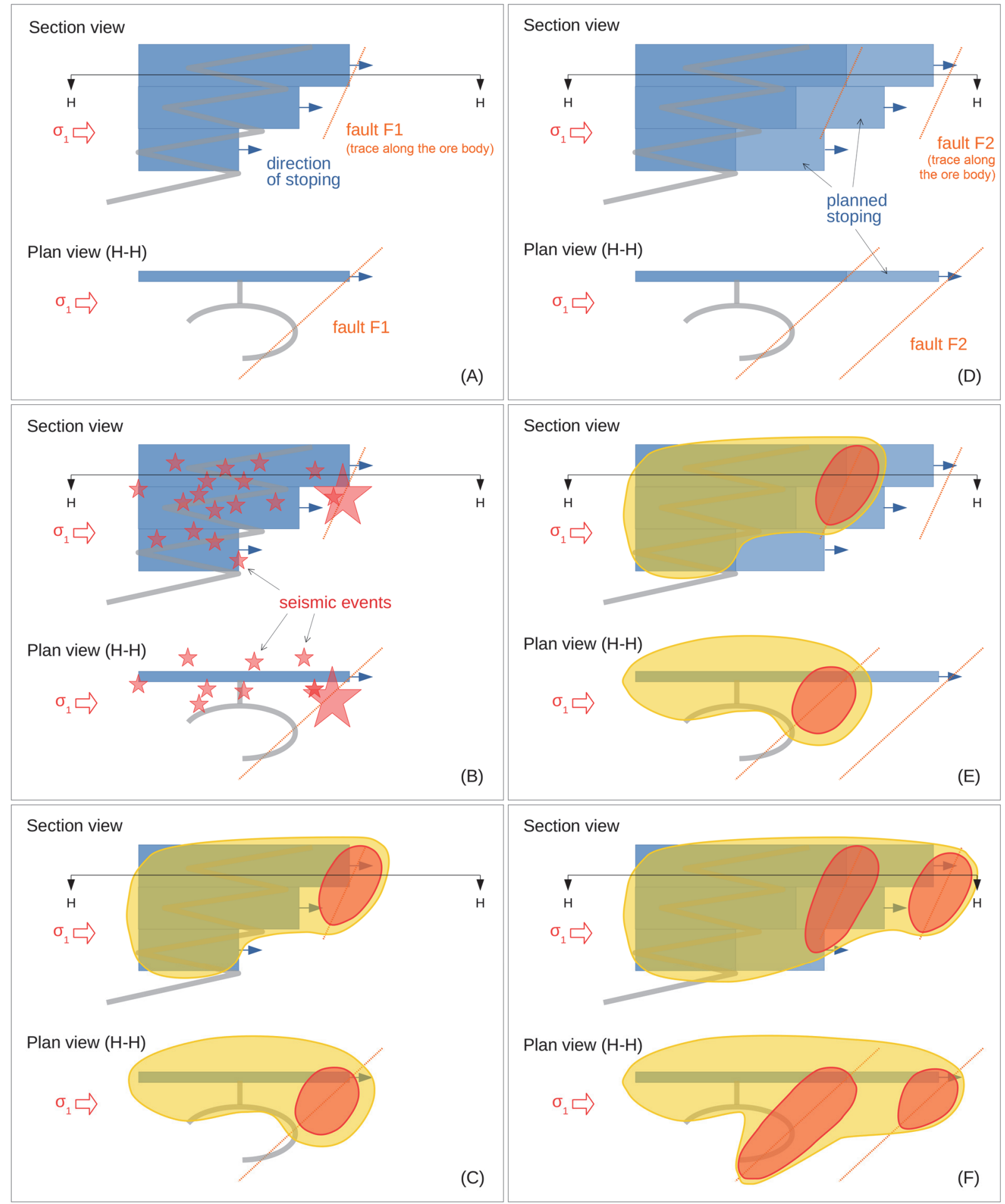

Figure 1 Cartoon diagrams illustrating the specifics of the assessment of seismic hazard in mines. Figures A-C show the current mining configuration, recorded seismic data and contours of seismic hazard calculated using this data. Figure $D$ shows the planned mining sequence and new information about geology (fault F2). The forecast of seismic hazard based on observed seismicity may not be relevant $(E)$ and the expected seismic response to future mining needs to be adopted in the calculation of hazard $(F)$ 
The sketch in Figure 1(a) shows an example of a steeply dipping narrow vein mining configuration with sub-horizontal maximum principal stress oriented parallel to the strike of the orebody. If the mine is equipped with a seismic monitoring system, then the recorded seismicity may look like that shown in Figure 1 (b). The smaller events on the sides of mined-out stopes can be related to the growth of yield zone, while the larger event close to the mining front may be interpreted as slip along the known fault F1, which is unclamped or has extra shear loading due to the approaching stoping. If the likelihood of potentially damaging seismic events is of concern for the short time interval into the future, then the hazard can be derived from the recorded seismicity. It presumes that near-term seismicity will be related to the yield zone around the stopes and the larger scale slip may re-occur on the fault F1. This logic will provide the contours of probabilities of occurrence of significant and large events shown in Figure 1(c).

These contours may not be relevant for longer time intervals, taking into account future mining (e.g. planned advancement of stopes shown in Figure 1(d)). It may be known that the mining front will approach another fault (F2), which has characteristics similar to F1. Although there were no significant seismic events associated with fault $F 2$ in the past, it may be reasonable to speculate that this fault will be unclamped during the execution of planned mining sequence and can deliver a large seismic event. It may also be considered that the extension of the mined-out span will result in the growth of the yield zone and fault F1 may be destabilised further away from the stopes (around the intersection with the decline). Taking these considerations into account, the projection of the forecast of seismic hazard based on recorded seismic data into longer time intervals in future (as shown in Figure 1(e)) may be incorrect, and the map of probabilities shown in Figure $1(\mathrm{f})$ will be more appropriate.

Two steps are required to deliver a forecast of seismic hazard for the planned mining in quantitative terms:

1. Model the changes of stresses and strains associated with future mining.

2. Transform these changes to the parameters of expected seismic events (location, time, size, mechanism).

A Salamon-Linkov approach to the modelling of expected seismicity based on the boundary element method was suggested in Malovichko and Basson (2014). The results presented later in this paper are obtained using this approach, however, it is not the only method to derive seismicity from numerical stress modelling. In general, any inelastic stress modelling code can be adopted for the assessment of expected seismicity. Considering that the modelled episodes of plastic strain increments occur suddenly, these can be transformed to seismic source parameters - time (mining step), location and seismic potency tensor, which can constitute a basic catalogue of seismic events.

The modelling of expected seismicity has to be calibrated; it needs to be shown that the recorded seismic response to historical mining can be replicated using a numerical model. The calibration typically includes running the numerical model for different variants of failure criteria or in situ stress fields. Having several variants of modelled seismicity may make it difficult to decide which one produces a better match to the observed data. There are multiple aspects of seismicity that can be focused on, including:

- Proximity of events to excavations (e.g. seismicity clustered close to stopes versus seismicity spread away from excavations).

- Relative position of events around excavations (e.g. clustering of events in the hanging wall and footwall versus association of events with pillars).

- Location of large seismic events (contours of cumulative seismic potency).

- Dominant source mechanisms in different parts of the mine (e.g. reverse faulting versus normal faulting).

- Orientation of principal axes of source mechanisms. 
It is useful to have a metric that quantifies the difference between the modelled forecasts and observed seismic data in terms of the above listed aspects. Such a metric will allow making a more objective selection of the best model at the calibration stage.

The seismic hazard calculated for future mining steps also needs to be tested against the observed seismicity after the planned mining is completed. The occurrence or absence of a single large seismic event in an area where high seismic hazard was forecast does not indicate that the forecast was successful or unsuccessful. Comparing the hazard forecasts with the spatial density of small-size events is also not a good strategy. Proper testing requires the analysis of multiple cases of significant seismic events and their relation to the corresponding hazard maps.

This paper illustrates that the same mathematical framework can be used both for the calibration and testing of seismic hazard forecasts. It is based on methods developed in crustal seismology in relation to the forecasting of tectonic earthquakes (Kagan \& Jackson 1994; Zechar 2010). The framework will be discussed in the next section and its application for calibration and testing of hazard forecasts will be illustrated in the second half of the paper.

\section{Method}

The forecast of seismic hazard includes several aspects of potentially damaging seismic events; these being their location, time, size (seismic potency) and source mechanism.

The temporal aspect of potentially damaging events is handled by the repetitive arrangement of the forecasting procedure. The forecasts are issued for specific time intervals and calibrated/tested using the seismic events recorded during those intervals.

Experience with the Salamon-Linkov method indicates that the size distribution of modelled seismic events is sensitive not only to the peak strength characteristics (e.g. static cohesion and friction angle), but also to the parameters describing post-peak behaviour (e.g. dynamic cohesion and friction angle, residual cohesion). Trying to adjust these parameters so that the potency frequency distribution of modelled events matches the observed data is currently a time-consuming procedure. At the current stage, it seems more robust to forecast seismic hazard using the potency frequency distribution based on observed data. The method of parametrisation of the potency frequency distribution, including the estimate of the upper cutoff $\left(P_{\max }\right)$ using the record theory, is described in Mendecki (2016). Therefore, in the suggested approach to the calibration and testing of seismic hazard forecasts, the potency of modelled seismic events is adjusted to match the observed potency frequency distribution (Malovichko 2017). Consequently, the size of events is not used in comparing and estimating the performance of forecasts.

The main focus of calibration and testing of forecasts is on two aspects of potentially damaging events:

1. Source locations.

2. Source mechanisms.

In this paper, the derivation of seismic hazard from the catalogue of seismic events is done using the procedure described in Malovichko (2017). This procedure considers the size of seismic events, i.e. large events have greater contribution to hazard compared to smaller events. There are other approaches to derive seismic hazard from seismic catalogues which are focused on the rate of seismic events (Wesseloo 2013).

The output of the procedure is a gridded spatial distribution $\operatorname{Pr}(\mathbf{x}, \mathrm{P}, \Delta \mathrm{V}, \Delta \mathrm{T})$ of probabilities of occurrence of events exceeding a specific potency $\mathrm{P}$ in elementary grid volumes $\Delta \mathrm{V}$ during time intervals $\Delta \mathrm{T}$. To take the possibility of 'surprise' events into account, the background probability was set to $0.1 \%$ of the peak probability in the entire volume, and then all gridded probabilities are adjusted to preserve the total probability.

The area skill score metric suggested in Zechar \& Jordan (2008) is used to assess the performance of the calculated hazard map $\operatorname{Pr}(\mathbf{x}, \mathrm{P}, \Delta \mathrm{V}, \Delta \mathrm{T})$. Figure 2 explains how the metric works in relation to the mining configuration and associated seismicity discussed in Figure 1 . The metric can be applied to a single forecast 
which implies that it will be compared with the uniform distribution of seismicity in space. It is more efficient to use the metric for comparing two forecasts ( $A$ and $B$ in Figure 2). For example, these can be forecasts based on different variants of the modelling of expected seismicity (calibration phase). Another option is that the forecast based on modelled seismicity is compared with the forecast based on observed seismic data (testing phase).
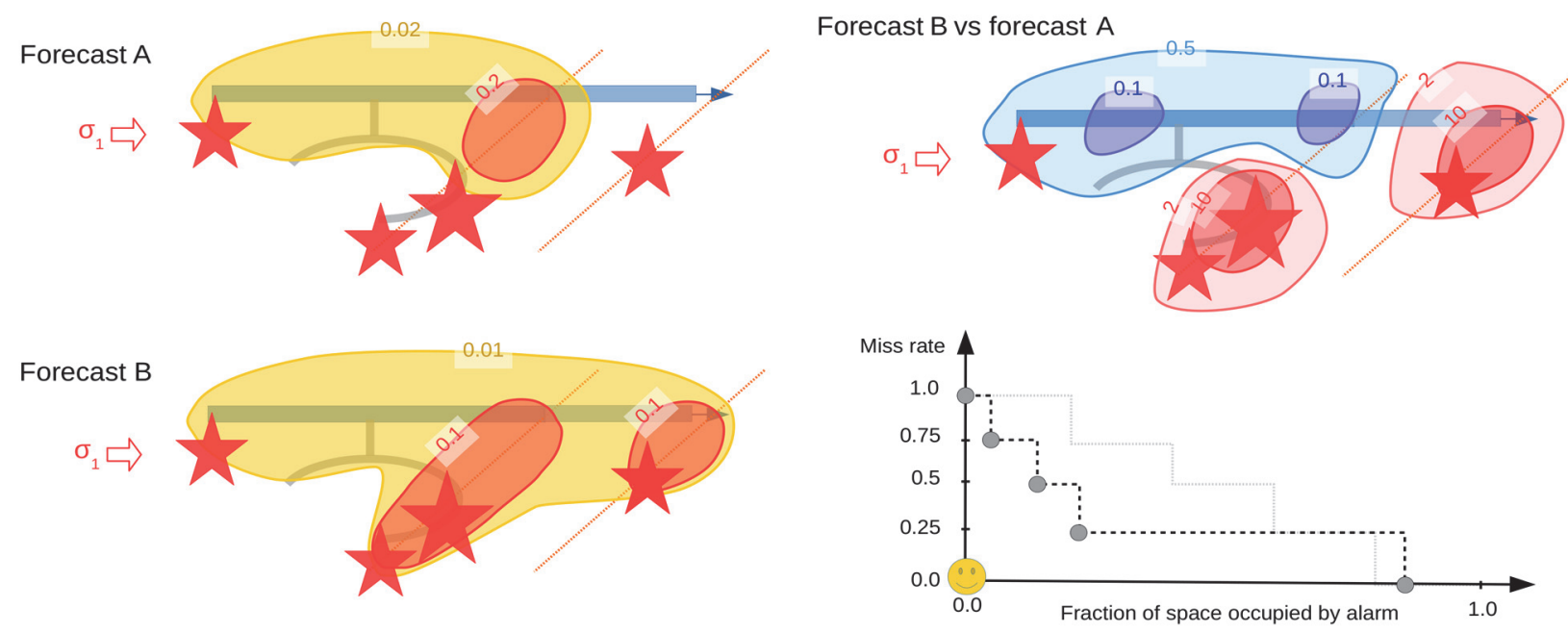

Figure 2 Cartoon illustrating the comparative testing of two forecasts of seismic hazard using an area skill score metric (suggested by Zechar \& Jordan 2008)

Potentially damaging events are used as target events in measuring the performance of the forecasts. Figure 2 shows four target seismic events as stars. Let's assume that these events are located in the same horizontal plane and there are two forecasts of seismic hazard ( $A$ and $B$ ) for this plane for the time interval of occurrence of target events (yellow and red contours on the left of Figure 2). The area skill score is assessed in the following way:

- The probability gain map, which describes the ratio of probabilities in forecast $B$ relative to forecast $A$, is calculated (top right in Figure 2).

- A specific level of probability gain is selected. The regions with higher probability (alarm volume) are identified and the number of target events located within these regions are counted. The size of alarm regions is expressed in terms of the fraction of total forecast volume (i.e. from 0 to 1 ) and the number of target events are described using the miss or hit rate (also from 0 to 1 ).

- The calculations are repeated from the highest to the lowest level of the probability gain, providing the trajectory in the so-called Molchan diagram with vertical axis describing the miss (or hit) rate and horizontal axis describing the fraction of space occupied by alarm (bottom right in Figure 2). If the trajectory passes close to the origin of the diagram (smile symbol), then forecast $B$ provides significant gain relative to forecast $A$. If the trajectory is located around the grey stairstep diagonal line, then forecast $B$ does not provide gain relative to forecast $A$.

- The area above the calculated trajectory represents an area skill score. It changes from 0 (bad performance of the forecast $B$ relative the forecast A) to 1 (excellent performance of the forecast $B$ relative the forecast $A$ ). The value of area skill score close to 0.5 indicates that both forecasts perform similarly.

In the cartoon example in Figure 2, three out of four target events are located in regions where the probability according to forecast $B$ is higher than for forecast $A$. Two of these events are plotted in localised regions where the probability in forecast $B$ is much higher than in forecast $A$, indicating a better predictive skill of forecast $B$. The overall measure of the performance of the forecast $B$ relative to $A$ (area skill score) will be around 0.6-0.7. 
The second aspect of testing the seismic hazard is related to source mechanisms. The expected source mechanism, moment tensor $\mathrm{M}_{\mathrm{ij}}(\mathbf{x}, \Delta \mathrm{V}, \Delta \mathrm{T})$, is calculated for each grid volume by averaging the normalised moment tensors of nearby seismic events. The coefficient of contribution of nearby events depends on the size and shape of their sources, as described in Malovichko (2017). The source mechanisms of target seismic events are compared with the expected mechanisms using the minimum rotation angle of double-couple (DC) components, as suggested in Kagan (2007). Table 1 illustrates the difference between mechanisms expressed in terms of this metric.

Table 1 Comparison of source mechanisms of events (adapted from Kagan 2007)

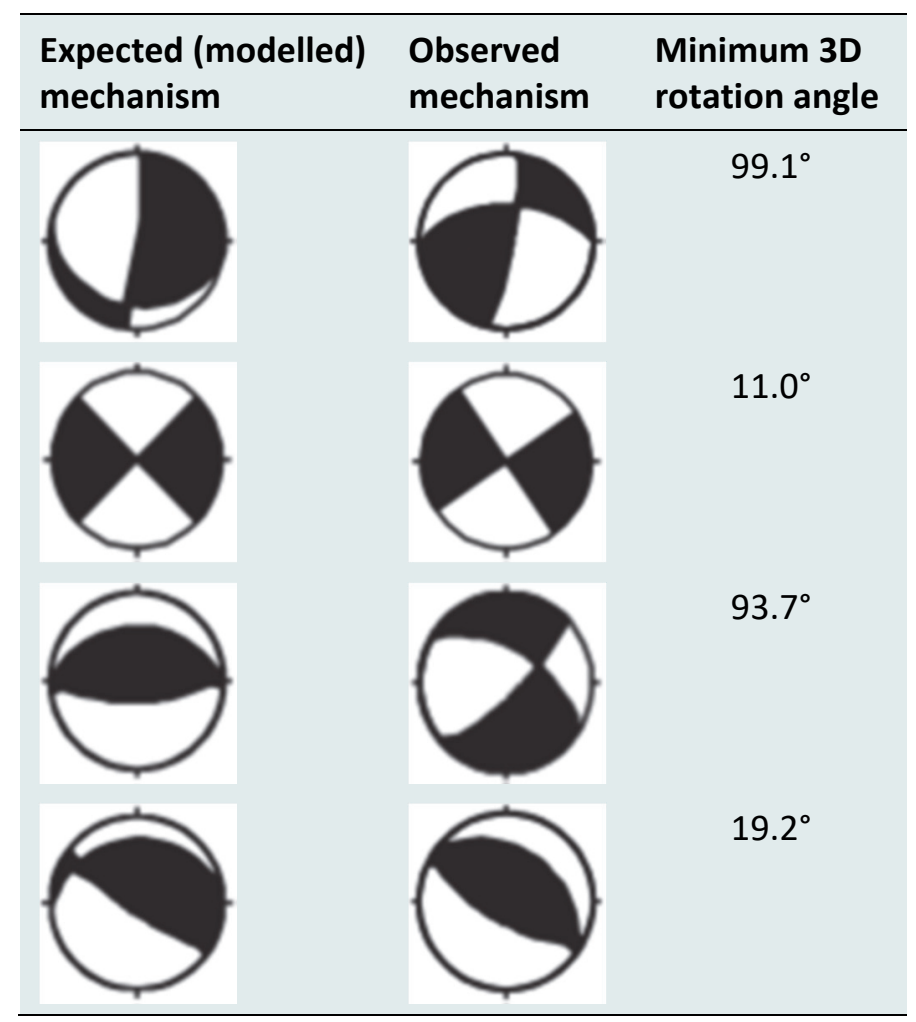

\section{Calibration of hazard forecasts}

There are two options for calibration:

1. Transform observed seismicity to hazard and test the modelled seismicity against it.

2. Transform the modelled seismicity to hazard and test the observed seismicity against it.

The first option may seem to be a better choice from a computational point of view as the hazard needs to be assessed only once. However, the second option is preferable for two reasons. Firstly, the forecast of seismic hazard for future mining sequences is based on modelled seismicity and it is therefore more natural to test hazard derived from modelled seismicity during the calibration phase. Secondly, some characteristics of modelled seismicity are more suitable for the derivation of the forecast. For instance, the modelling prescribes the geometrical characteristics of seismic sources, which allows the associated hazard to be distributed in space appropriately. The observed seismic data typically lacks information about the orientation and dimension of the sources of large events, therefore their contribution to seismic hazard is isotropic (spherically symmetric) in space, which is not very physical.

Information from a narrow vein mine in Australia is used for the illustration of hazard calculations in this paper. Figure 3 shows the mining configuration and stoping sequence starting from July 2011. The stoping sequence was split in to 29 quarterly steps: 25 steps (from 2011 Q3 to 2017 Q3) were used for calibration and the most recent four steps (from 2017 Q4 to 2018 Q3) were used for testing, described in the next section. 


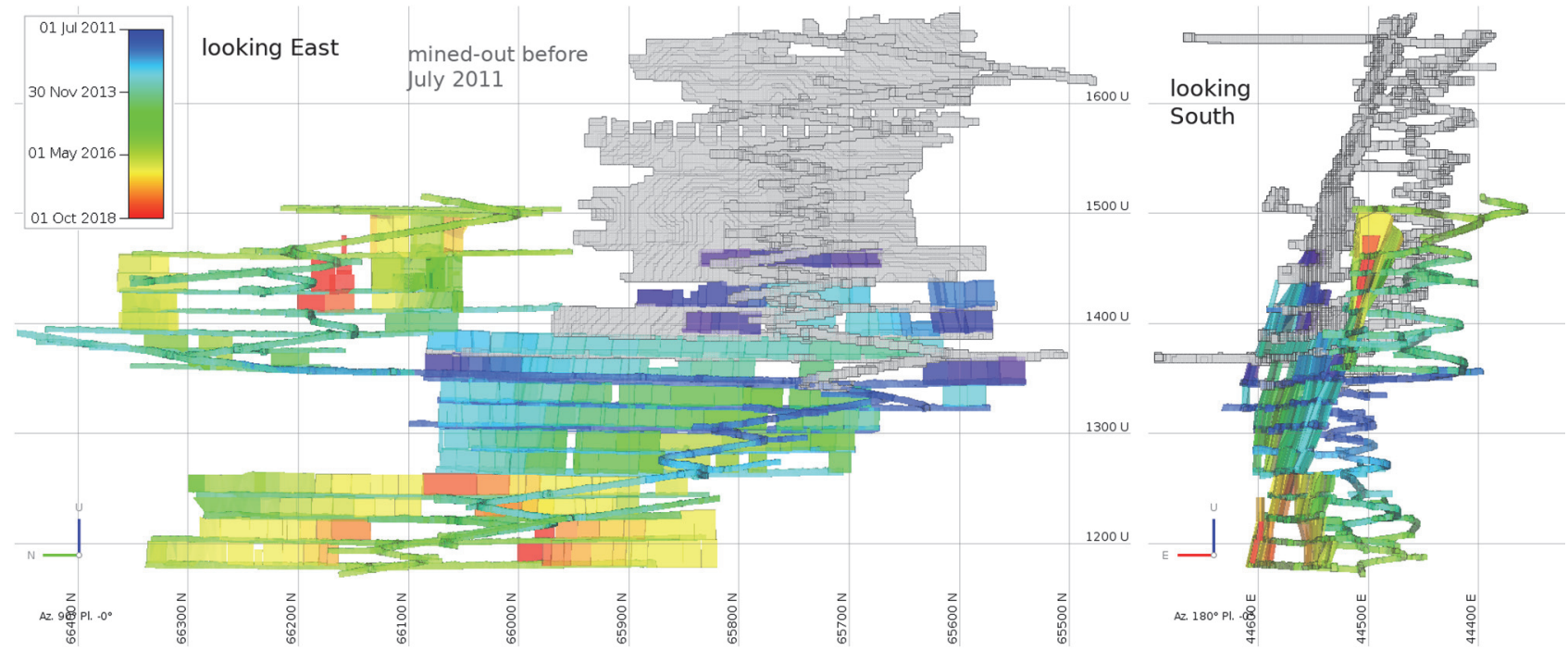

Figure 3 Mining sequence from July 2011 to September 2018. The mined-out stopes are coloured according to quarterly steps. The development drives are sampled using the coarser steps

The modelling of seismicity was done for three variants of input parameters - in situ stress and failure criteria listed in Table 2. Variants $A$ and $B$ have sub-horizontal maximum principal stress striking approximately parallel to the orebody. In variant $C$, the in situ stress field was rotated $90^{\circ}$ and the maximum principal stress was oriented sub-orthogonal to the orebody. The difference between variants $A$ and $B$ is that a weaker joint set (FW North) was introduced in the latter case.

\section{Table 2 Modelling parameters}

\begin{tabular}{|c|c|c|}
\hline Model & In situ stress & Failure criteria \\
\hline A & $\sigma_{1}$ along the orebody ${ }^{1}$ & $\mathrm{MC1}^{3}$ for rock mass \\
\hline B & $\sigma_{1}$ along the orebody & $\begin{array}{l}\text { MC1 for rock mass, } M C 2^{4} \text { for the FW North joint set } \\
\text { (dip/dip direction }=50^{\circ} / 130^{\circ} \text { ) }\end{array}$ \\
\hline C & $\sigma_{1}$ across the orebody ${ }^{2}$ & MC1 for rock mass, MC2 for the FW North joint set \\
\hline
\end{tabular}

The results of modelling of seismicity for calibration time intervals are shown in Figure 4 along with the observed data. Inclusion of the weaker joint set in variants B and C facilitates the development of yield zones around the stopes, therefore the modelled seismicity is distributed in a larger volume compared to variant A. Changing the orientation of in situ principal stresses also affects the location of events. In variants $A$ and $B$, seismic events are mostly distributed in the hanging wall and footwall of mined-out stopes, while in variant C many events are attributed to the abutment of stopes and pillars.

Note that all three considered models are not perfect. For example, they all underestimate wide-spread clustering of seismicity in the hanging wall. 


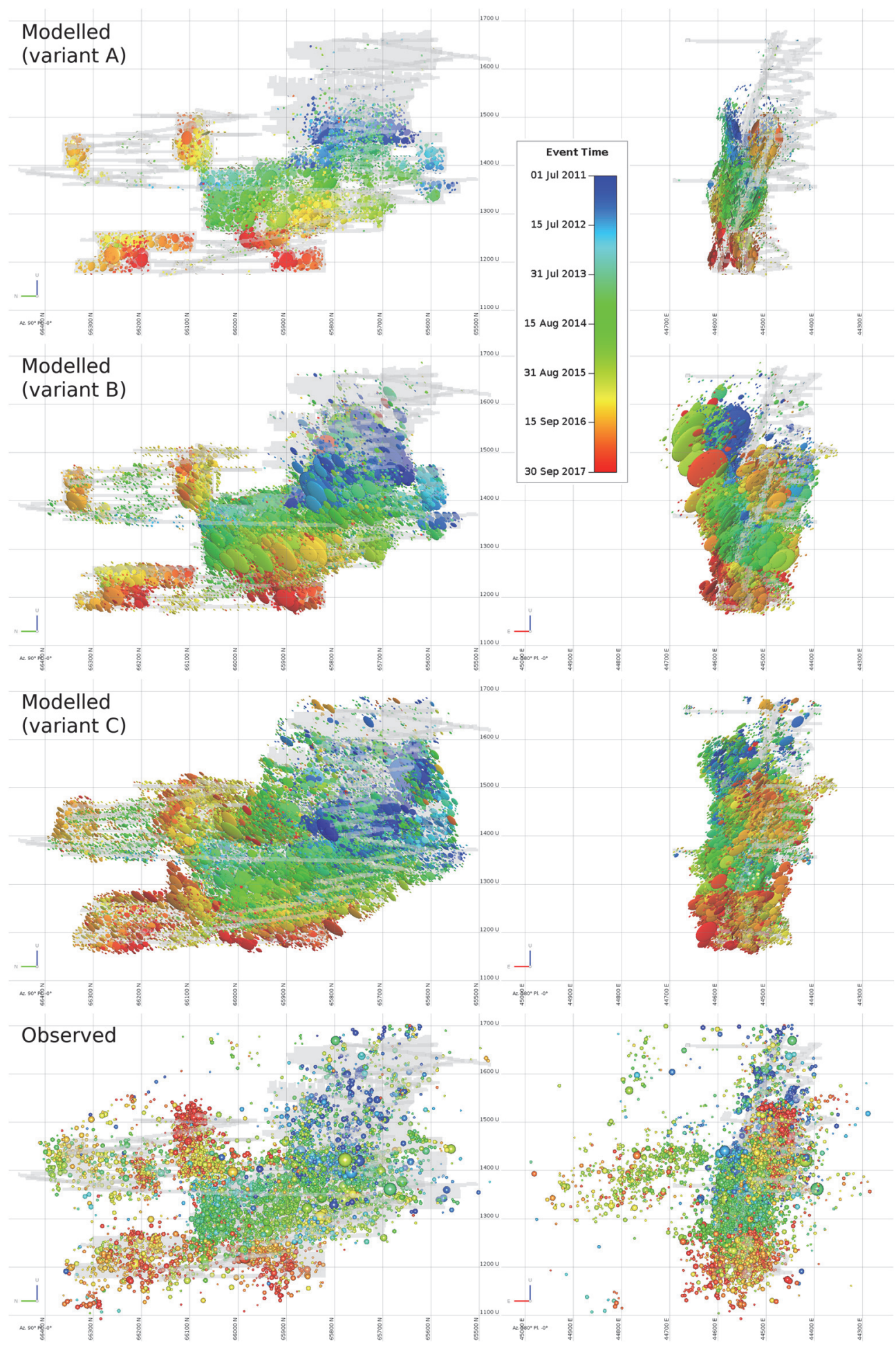

Figure 4 Modelled and observed seismicity for calibration period (July 2011 to September 2018). The size and orientation of sources of modelled events are shown using ellipsoids. The observed seismic events are shown as spheres sized according to logP

The results of the transformation of modelled seismicity to seismic hazard using the approach described in Malovichko (2017) are shown in Figure 5. The isosurfaces describe the probability of occurrence of seismic events with $\log \mathrm{P} \geq-0.6$ (corresponding to Hanks-Kanamori moment magnitude $\mathrm{m}_{\mathrm{HK}} \geq 0.5$ ) within $\Delta \mathrm{V}=30 \times$ $30 \times 30 \mathrm{~m}$ volume during $\Delta \mathrm{T}=2,284$ days (or six years and three months - calibration period): $20 \%, 4 \%$ and $1 \%$ for red, green and blue isosurfaces, respectively. 
The double-couple component of the dominant source mechanisms for each grid cell is shown using a beach ball symbol with red P-axis.

The significant events of interest for forecasting are events with $\log \mathrm{P} \geq-0.6\left(\mathrm{~m}_{\mathrm{HK}} \geq 0.5\right)$, having predominantly deviatoric mechanisms (less than $35 \%$ isotropic component). There were 14 such events recorded during the calibration period (2011 Q3 - 2017 Q3), and these are plotted in the bottom of Figure 5.

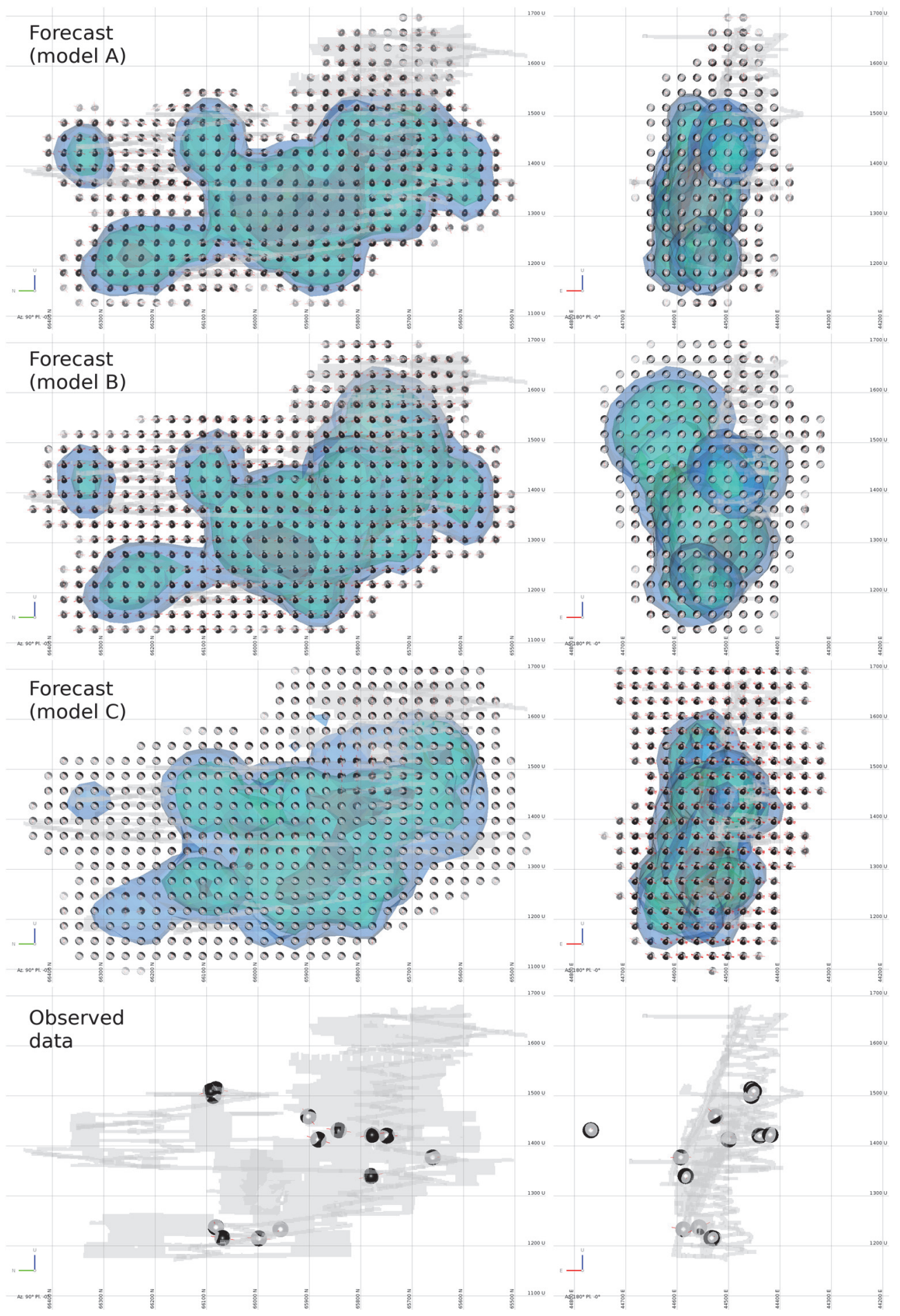

Figure 5 Seismic hazard maps for the variants of modelled seismicity in Figure 4. The isosurfaces characterise the probability of occurrence of significant events and the gridded beach ball symbols describe the expected source mechanisms. The significant seismic events with deviatoric mechanisms recorded during the calibration period are shown using beach balls in the bottom 
The Molchan diagrams and area skill scores were calculated for pairs of forecasts (model A relative to model B, model A relative to model C, etc.) Three of these are shown in Figure 6. For example, the first plot in Figure 6 shows that eight significant events occurred in the localised regions with higher probability in model $B$ relative to model $A$ and these regions occupy less than $5 \%$ of the entire space. At the same time there were five significant events that occurred in the regions where the probability in model B was lower. These regions are also quite localised, occupying less than $5 \%$ of the entire space. The overall measure of the performance of forecast $B$ relative to forecast $A$ in terms of area skill score is 0.679 .
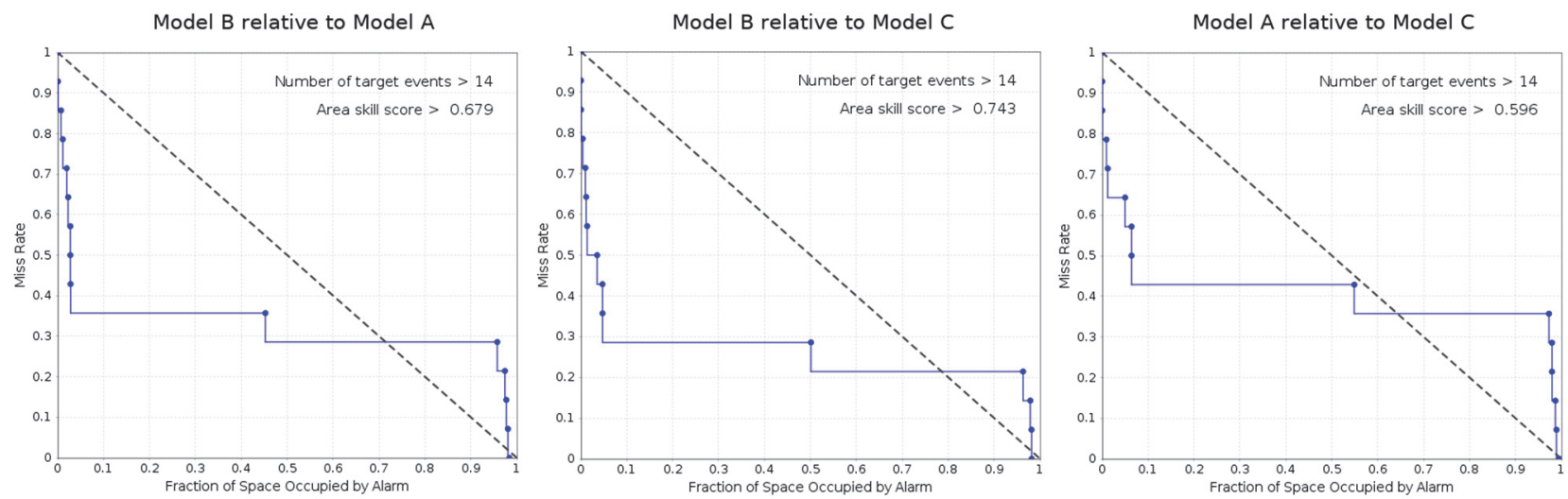

Figure 6 Molchan diagrams for the 14 recorded significant events and three variants of forecast (models $A, B$ and $C$ ). The results for only three combinations out of six forecast pairs are presented

The scores for all pairs of forecasts are listed in Table 3. The results show that model B predicted the location of significant seismic events of interest better than models $A$ and $C$.

Table 3 Results of calibration

\begin{tabular}{lll}
\hline Model & Match of location - area skill score & Match of mechanisms $-3 D$ rotation angle \\
\hline A & 0.462 relative to model B & Mean angle $=62^{\circ}$, median angle $=61^{\circ}$ \\
& 0.596 relative to model C & \\
B & 0.679 relative to model A & Mean angle $=48^{\circ}$, median angle $=41^{\circ}$ \\
& 0.743 relative to model C & \\
C & 0.547 relative to model A & Mean angle $=75^{\circ}$, median angle $=86^{\circ}$ \\
\hline
\end{tabular}

The minimum 3D rotation angles between the mechanisms of observed significant events and expected mechanisms are presented in Figure 7. The calculations are done not only for the hypocentres of events, but also for the neighbouring areas according to the spatial kernel function used for the gridding. This is why the histograms in Figure 7 count the number of gridded cells affected by significant events and not the number of significant events. The mean and median 3D rotation angles are quoted in the histograms and listed in Table 3.

Models A and B provide much better prediction of the mechanisms compared to model C. This makes sense as the expected mechanisms correlate with the in situ stress field assumed in the models. The orientation of $\sigma_{1}$ along the orebody in models $A$ and $B$ results in the expectation of strike-slip and reverse faulting mechanisms with $\mathrm{P}$-axis also trending along the orebody (Figure 5 ). The assumption that in situ $\sigma_{1}$ is oriented across the orebody in the model $\mathrm{C}$ results in the expectation of mechanisms with $\mathrm{P}$-axis oriented sub-normal to the orebody (Figure 5). The source mechanisms of recorded significant events (bottom of Figure 5) generally have P-axes oriented along the orebody, which correlates with the predictions of models A and B. 

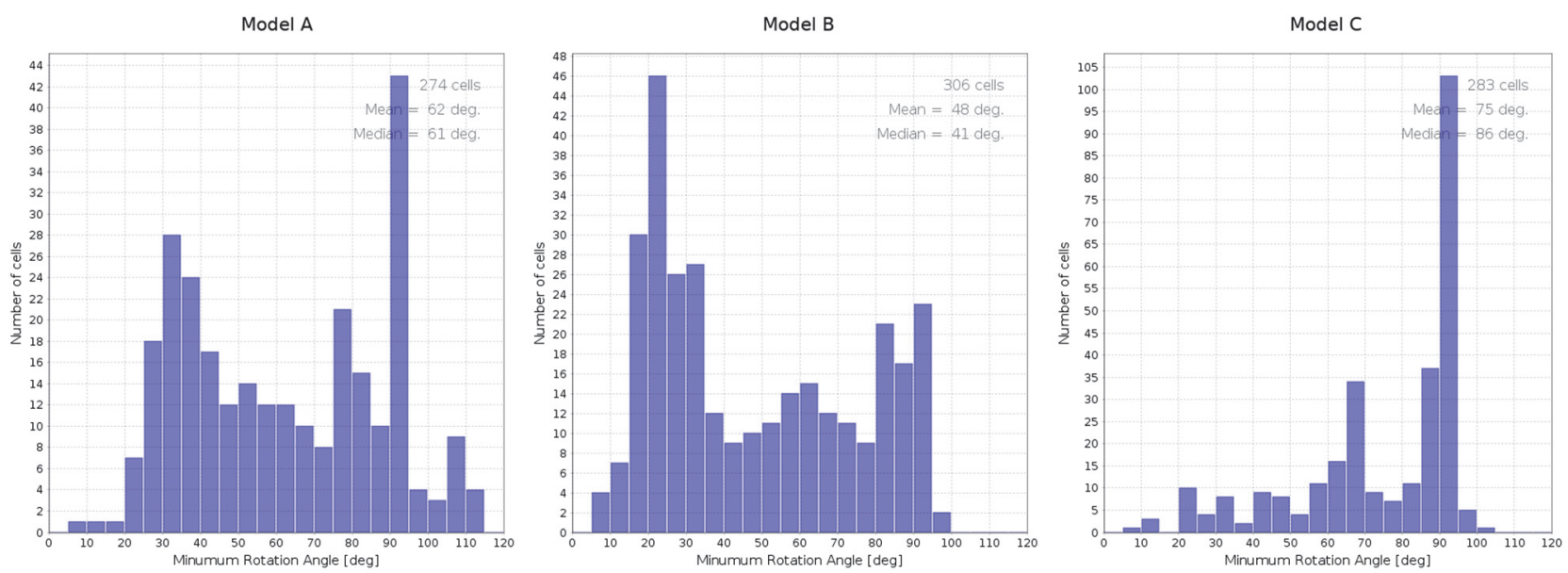

Figure 7 Distributions of angles of rotation from the mechanisms of the 14 observed significant seismic events to expected mechanisms for three variants of the forecast (models $A, B$ and $C$ )

Considering both the spatial and source mechanism aspects of forecasts, better predictive skills are demonstrated by model $\mathrm{B}$ compared to models $\mathrm{A}$ and $\mathrm{C}$. This justifies the selection of this model for forecast of seismic hazard in future.

\section{$4 \quad$ Testing of hazard forecasts}

Retrospective testing of the forecast is illustrated using the time interval from 1 October 2017 to 1 October 2018 which was excluded from the calibration. The forecast of hazard was derived from the combination of observed seismic data from 1 July 2011 to 1 October 2017 and expected seismicity for 1 October 2017 to 1 October 2018, obtained using model B described in the previous section. Figure 8 shows the isosurfaces of probability of occurrence of seismic events with $\log \mathrm{P} \geq-0.6\left(\mathrm{~m}_{\mathrm{HK}} \geq 0.5\right)$ within $\Delta \mathrm{V}=30 \times 30 \times 30 \mathrm{~m}$ volume during $\Delta T=365$ days: $10 \%, 3 \%$ and $1 \%$ for red, green and blue isosurfaces, respectively. The beach balls characterise the deviatoric component of expected source mechanisms.

There were nine seismic events with $\log P \geq-0.6$ and deviatoric source mechanisms recorded between 1 October 2017 to 1 October 2018 (bottom of Figure 8). Most of these are located around the areas of mining undertaken during the testing time interval.

The performance of the forecast in space was assessed relative to the forecast based only on observed seismic data recorded from 1 July 2011 to 1 October 2017 (i.e. forecast assuming that tomorrow will be the same as today). The Molchan diagram on the left of Figure 9 shows that five out of nine target events plot in localised regions (less than a few percent of the total volume), where the probabilities calculated based on the combination of observed and modelled data are higher than the probabilities calculated using only observed data. The area skill score is 0.68 , which indicates that the modelled seismicity provides significant gain to the forecast.

The DC component of the mechanisms of target events have mean and median 3D rotation angles of $52^{\circ}$ and $56^{\circ}$, respectively, from the expected DC mechanisms. The mean value is lower than the expected value of random 3D rotations of DC mechanisms, which is approximately $78^{\circ}$ (Kagan 1992). 


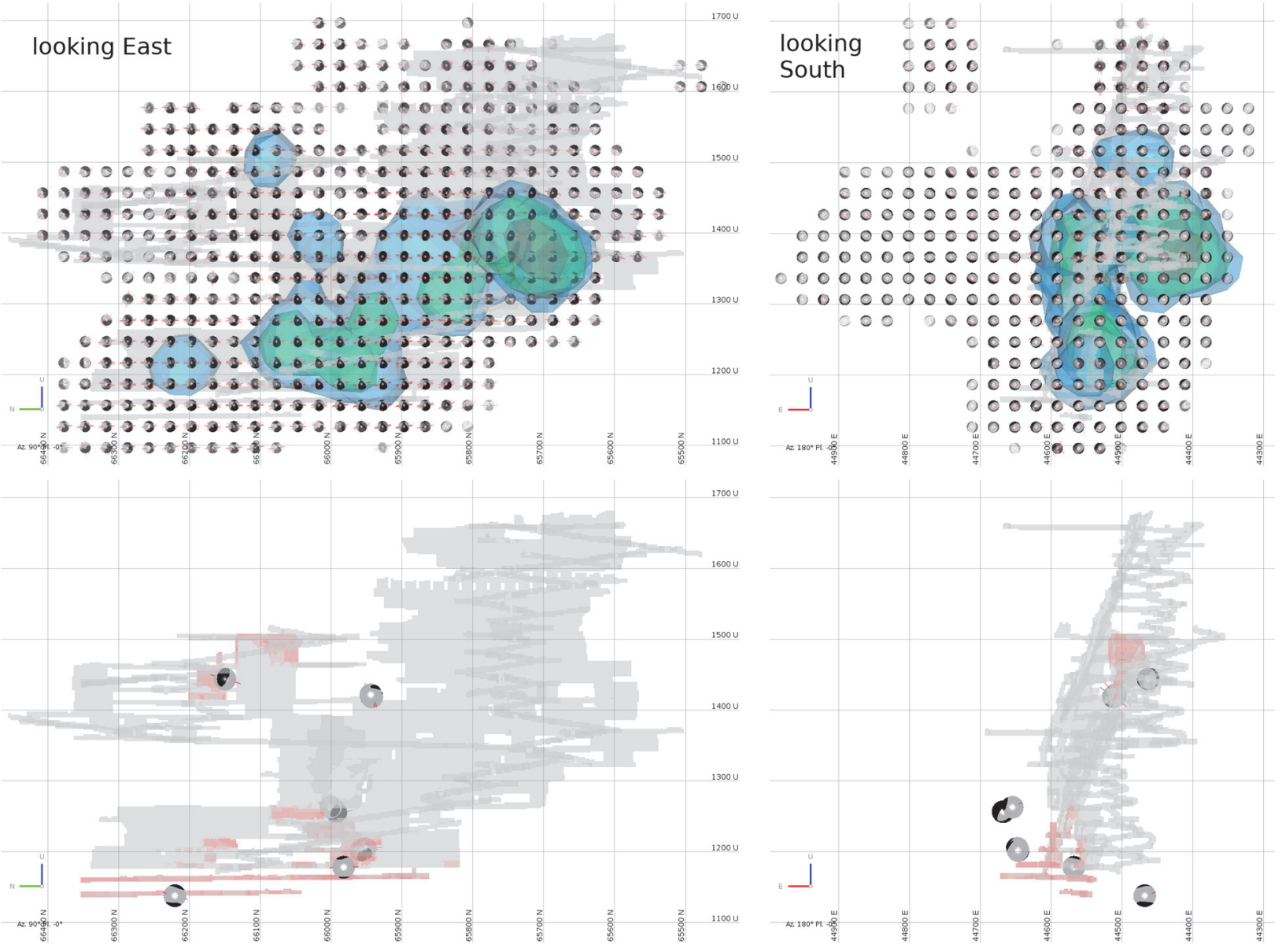

Figure 8 Seismic hazard map based on the combination of seismic data recorded from 2011 Q3 to 2017 Q3 and modelled seismicity for 2017 Q4 - 2018 Q3 (top). Significant seismic events with deviatoric mechanisms recorded during the testing period (2017 Q4 - 2018 Q3) are shown using beach balls (bottom). The red wireframes show mined-out volumes during the testing period
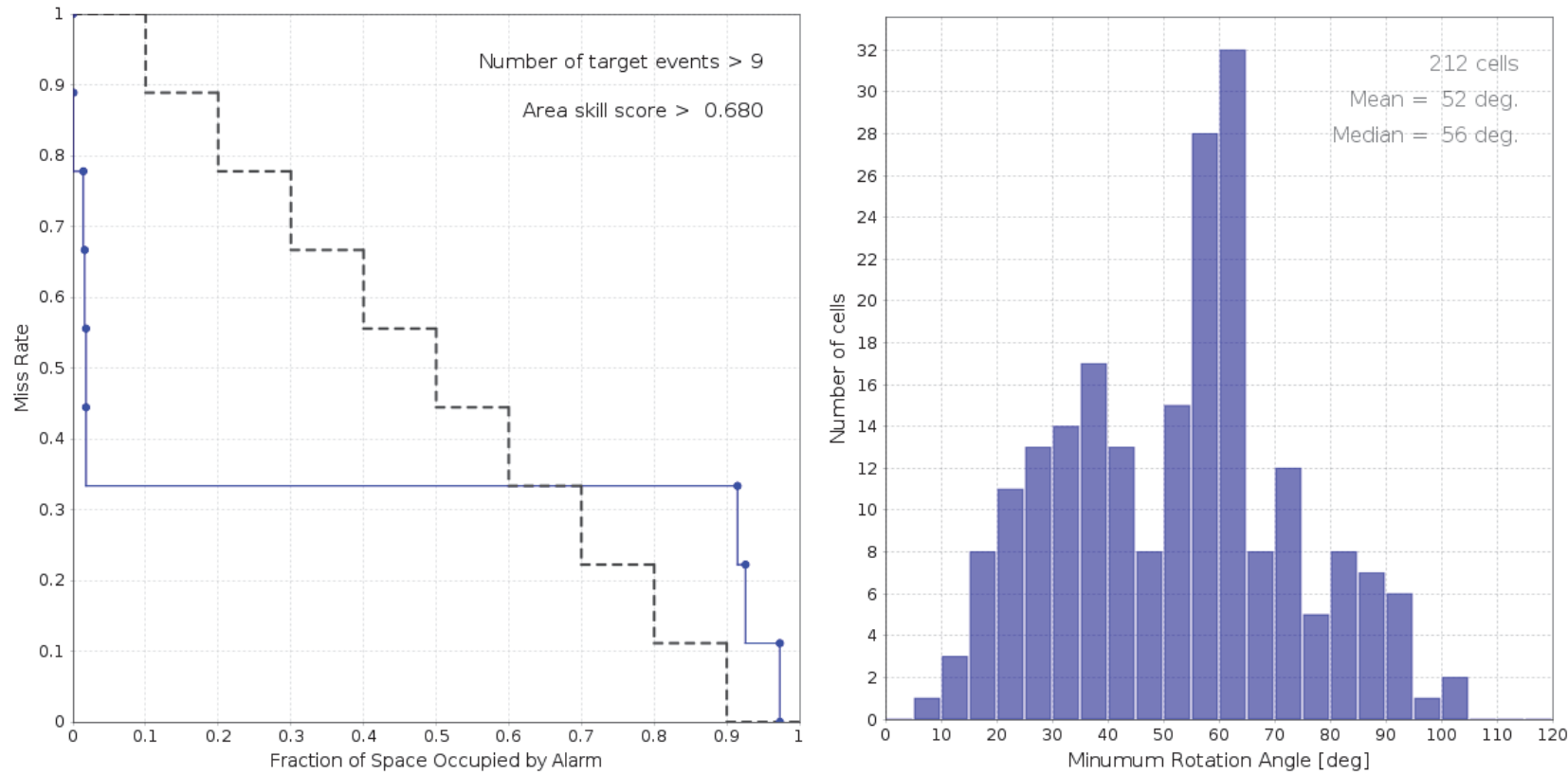

Figure 9 Results of retrospective testing of seismic hazard forecast for 2017 Q4 - 2018 Q3 based on the combination of observed and modelled data 


\section{Discussion and conclusion}

The paper discusses the calibration and testing of two aspects of seismic hazard in mines; location of potentially damaging seismic events and their source mechanisms. The spatial aspect of hazard seems to be more important from a practical point of view. However, there are benefits to forecasting the mechanisms of events as well. Firstly, the source mechanisms are sensitive to in situ stress and therefore, provide constraints to numerical models used to derived expected seismicity during the calibration stage. Secondly, differences in expected seismic source mechanisms may be of interest in relation to the assessment of associated damage to excavations. In this paper, the main focus of the forecast was on slip-type events which have deviatoric source mechanisms and may affect the excavation both in terms of permanent deformation and strong ground motion. Future calibration and testing of forecasts may be potentially extended to crush-type seismic events which represent episodes of sudden volumetric inelastic deformation (e.g. strainbursting, pillar failure).

Various metrics were developed in crustal seismology to test the forecasts of tectonic earthquakes. Some of them are used on a routine basis (Schorlemmer et al. 2018). This paper suggests the adoption of one of these metrics - area skill score - for testing the spatial forecasts of seismic hazard in mines. With regards to forecasts of earthquake mechanisms, it was suggested to test the rate of events in discretised a 3D domain of focal mechanisms (described by orientations of $\mathrm{P}$ - and T-axes) in a similar way to what is done for the discretised domain of source locations, origin times and magnitudes (Schorlemmer et al. 2007). However, according to the author's knowledge, this approach has not yet been applied to real data. This paper utilises a simple metric to quantify the agreement between the observed and expected source mechanisms (minimum 3D rotation angle of DC mechanisms) and does not involve the treatment of uncertainties. The latter aspect requires further development.

Seismically-active mines typically have seismic hazard management plans. Managing the hazard implies that it first needs to be assessed, i.e. the mine has to document the expectation regarding potentially damaging seismic events in the near future (What is the likelihood of potentially damaging events? Where are they expected to happen?). Although it becomes customary to assess size distribution hazard (likelihoods of large events for seismogenic regions, which typically comprise the entire mine), documenting the expected distribution of seismic hazard in space is done very infrequently. A qualitative assessment is presented sometimes (e.g. maps of modelled concentrations of stresses expected in future) without referring to probabilities or likelihoods of potentially damaging events. There are several benefits in conducting proper seismic hazard assessment and its ongoing testing:

- Seismic hazard delivered in quantitative terms can be objectively compared with other hazards and managed using the risk assessment procedures established at the mine.

- Performance of hazard assessment can be monitored over time. For example, degradation of the area skill score or increase of the minimum 3D rotation angle of DC mechanisms indicates that the forecasting procedures need to be revisited.

- Improvement of forecasts can be guided. For example, seismic events deteriorating the performance of the forecast both in terms of location and source mechanisms can be detected (e.g. events contributing to the parts on the right of plots in Figure 9) and these can prompt adjustments of the input parameters of the numerical stress models utilised in the derivation of expected seismic response (e.g. orientation of in situ stress, failure criteria).

\section{Acknowledgement}

Renison mine kindly permitted the use of the data. I am grateful to the senior geotechnical engineer of the mine, Kevin Stacey, for ongoing support and interest to the seismic hazard assessment and testing work. 
Stephen Meyer from the Institute of Mine Seismology (IMS) undertook constructive review of the paper. Ernest Lötter and Gys Basson (both from IMS) assisted with the implementation of the calculation and visualisation of seismic hazard. Their help is much appreciated.

\section{References}

Field, EH 2007, 'Overview of the working group for the development of Regional Earthquake Likelihood Models (RELM)', Seismological Research Letters, vol. 78, no. 1, pp. 7-16.

Kagan, YY 1992, 'Correlations of earthquake focal mechanisms', Geophysical Journal International, vol. 110, pp. 305-320.

Kagan, YY \& Jackson, DD 1994, 'Long-term probabilistic forecasting of earthquakes', Journal of Geophysical Research, vol. 99, no. B7, pp. 13685-13700.

Kagan, YY 2007, 'Simplified algorithms for calculating double-couple rotation', Geophysical Journal International, vol. 171, pp. 411-418.

Malovichko, D \& Basson, G 2014, 'Simulation of mining induced seismicity using Salamon-Linkov method', in M Hudyma \& Y Potvin (eds), Proceedings of the Seventh International Conference on Deep and High Stress Mining, Australian Centre for Geomechanics, Perth, pp. 667-680.

Malovichko, D 2017, 'Assessment and testing of seismic hazard for planned mining sequences', in J Wesseloo (ed.), Proceedings of the Eighth International Conference on Deep and High Stress Mining, Australian Centre for Geomechanics, Perth, pp. 61-77.

Mendecki, AJ 1997, Seismic Monitoring in Mines, Chapman and Hall, London, p. 262.

Mendecki, AJ 2016, Mine Seismology Reference Book: Seismic Hazard, Institute of Mine Seismology, 88 p.

Schorlemmer, D, Gerstenberger, MC, Wiemer, S, Jackson, DD \& Rhoades, DA 2007, 'Earthquake likelihood model testing', Seismological Research Letters, vol. 78, no. 1, pp. 17-29.

Schorlemmer, D, Werner, MJ, Marzocchi, W, Jordan, TH, Ogata, Y, Jackson, DD, Mak, S, Rhoades, DA, Gerstenberger, MC, Hirata, N, Liukis, M, Maechling, P, Strader, A, Taroni, M, Wiemer, S, Zechar, JD \& Zhuang, J 2018, 'The collaboratory for the study of earthquake predictability: achievements and priorities', Seismological Research Letters, vol. 89, no. 4, pp. $1305-1313$.

Wesseloo, J 2013, 'Towards real-time probabilistic hazard assessment of the current hazard state for mines', in A Malovichko \& D Malovichko (eds), Proceedings of the Eighth International Symposium on Rockbursts and Seismicity in Mines, Geophysical Survey of Russian Academy of Sciences, Obninsk, and Mining Institute of the Ural Branch of the Russian Academy of Sciences, Perm, pp. 307-312.

Zechar, JD \& Jordan, TH 2008, 'Testing alarm-based earthquake predictions', Geophysical Journal International, vol. 172, pp. 715-724.

Zechar, JD 2010, Evaluating Earthquake Predictions and Earthquake Forecasts: A Guide for Students and New Researchers, Community Online Resource for Statistical Seismicity Analysis, viewed 25 November 2018, http://www.corssa.org/articles/theme_6 